\title{
Assesment of Some Udder and Teat Traits of Honamli Goats in Terms of Dairy Characters
}

\section{Aykut Asım AKBAȘ ${ }^{1 *}$, Özkan ELMAZ ${ }^{1}$, Mehmet SARI $^{2}$, Mustafa SAATCI $^{3}$}

\author{
1Department of Animal Science, Faculty of Veterinary Medicine, Mehmet Akif Ersoy University, Burdur \\ 2Department of Animal Science, Faculty of Agriculture, Ahi Evran University, Kırşehir \\ 3Department of Animal Science, Faculty of Agriculture, Muğla Sitkı Koçman University, Muğla
}

Received 04.08.2019 Accepted 20.10.2019

\begin{abstract}
Study was conducted to evaluate the dairy characters of Honamlı goats by using ADGA scoring system. For this aim, eight Honamlı goat flocks reared under local breeder condition in Antalya and Burdur provinces were used. Five goats from each flock totally 40 goats with the highest score were defined. Some udder and teat characteristics of these goats were determined during six months (March-August). While Honamlı goats would be given in the acceptable category for dairy character with their numerical final scores (70-79); relatively higher values were detected from the flocks in Antalya province than Burdur ones $(\mathrm{P}<0.05)$. The udder characteristics increased in April based on course of lactation of Honaml goats in the best flocks (Antalya province) were determined as follows, udder depth, udder circumference, udder width, teat length, teat diameter, the distance between teats and the distance to floor from the teat $21.73 \mathrm{~cm}, 46.97 \mathrm{~cm}, 17.06 \mathrm{~cm}, 8.05 \mathrm{~cm}, 3.08 \mathrm{~cm}$, $14.47 \mathrm{~cm}$ and $37.22 \mathrm{~cm}$, respectively.

It was thought that results of study were important to be an introduction for following studies that is going to be potential goats having dairy characteristics among Honamli goats.
\end{abstract}

Keywords: goat, Honaml, udder, teat, dairy character

\section{Introduction}

Mankind has been evaluating the milk of goats for approximately 11 thousand years now. ${ }^{1}$ Having an important place in Anatolian cultural and social life, goat breeding also takes place on land generally unsuitable for agriculture and Livestock. ${ }^{2}$ Honamlı goats, which are named after the Honamlı nomads, are considered as one of a native goat breed that reared for their meat, milk, and wool. ${ }^{3}$ They officially registered by Turkish Ministry of Food, Agriculture and Livestock as an orginal goat breed in the year of 2015. ${ }^{4}$ Recently, there has been an obvious increase in the demand for goat milk and products in developed and developing countries due to its nutritional value and economic importance.

There are many factors affecting milk yield in goats. These factors involve breed, age, feeding, body structure, live weight, and udder structure. ${ }^{5}$ In some studies, it is reported that milk yield is affected positively by live weight, ${ }^{6}$ as well as udder and teat characteristics especially in dairy goats. $^{7}$

Body sizes that are used for identifying the animals numerically, forming an opinion about their body structure and determining their breed characteristics show an alteration seasonally and are under the effect of factors like age, gender, delivery method, genotype and feeding style. Similarly, the process of determining body sizes which also includes the comparison of breeds and animals from the same breed is important for finding the early selection cri-

\footnotetext{
* Corresponding author: Aykut Asım AKBAŞ Tel: +90248 2132075; fax: +90248 2132000 icould_akbas@hotmail.com
} 
teria for certain yields and improving the animals. ${ }^{8,9}$ Phenotypic evaluations aimed at determining future breeding animals in terms of required yields were applied frequently in the 1960s. ${ }^{10}$ In addition, a linear scoring system has started to be used since the beginning of the $1980 \mathrm{~s} .{ }^{11}$ Linear evaluations which contain individual evaluation of animals to reveal their yield potential for genetic progress ${ }^{12}$ to be conducted with selection enable to reach higher levels of heredity compared to subjective evaluation. ${ }^{13}$

The aim of the current study was to investigate some udder and teat traits such as udder depth, udder circumference, udder width, teat length, teat diameter, distance between teats and distance to floor from the teat of Honamli goats in terms of dairy characters.

\section{Material and Methods}

\section{Animals and Data Collection}

The present study was carried out in Honamlı goat flocks reared under local breeder condition in Antalya and Burdur provinces. So, no additional care or feeding conditions were provided to the animals. At the beginning of this study, a detailed survey was done to determine Honamlı goats having a different udder structure, deeper chest, long and thin legs and thus, displaying dairy characteristics especially in some of the Honamli goat flocks reared by the local breeder condition. So, the eight Honamli goat flocks were detected and then five goats from each flock totaly 40 goats with 2 years old age and having the first pregnancy with the highest score according to the scoring in terms of general apperance, dairy character, body capacity and mammary by applying ADGA score card were defined. For eliminating and also avoid the potential effects of factors such as age, lactation period, birth type etc., the goat at the same case were chosen for udder and teat measurements.

In the study, some the udder and teat characteristics such as udder depth, udder circumference, udder width, teat length, teat diameter, the distance between teats and the distance to floor from the teat ${ }^{14}$ of these goats were determined during six months (March-August).

The study was approved by the Mehmet Akif Ersoy University Local Ethics Committee on Animal Experiments (26.09.2014, resolution number: 99).

\section{Statistical Analyses}

All statistical analyses were carried out using Minitab 16.1 statistical package. ${ }^{15}$ The normality test was applied to data and also the coefficient of variation was calculated between 18-20. According to that the data were considered having normal distribution with acceptable heterogeneity. Oneway ANOVA was used to determine the differences between flocks on udder and teat traits. Tukey analysis was used to control for significance of differences between subgroups $(\mathrm{P}<0.05)$. Therefore, two sample $\mathrm{T}$ Test was applied in order to compare flocks separeted for provinces.

\section{Results}

During stages of determining the herds containing dairy animals within the scope of the study, it was observed that there were animals with many different udder structures in Honamli goat flocks reared by the locals, which shows a parallelism with the aforementioned literature. According to the interviews conducted with breeders and the observations of the study team, it was suggested that the aforementioned udder structures could be collected under three basic categories (Figure 1).

In the study, Honamli goats would be given in the acceptable category for dairy character with their numerical final scores (70-79); relatively higher values were detected from the flocks in Antalya province than Burdur ones (Table 1). Tables (2-7) show some udder and teat sizes of goats determined within the scope of the study. Among the relevant characteristics; udder depth, udder circumference and udder width of goats in Antalya (first four flocks) and Burdur (last four flocks) for March were determined respectively as $21.62 \mathrm{~cm}, 46.80 \mathrm{~cm}$ and $16.95 \mathrm{~cm}$ and $21.40 \mathrm{~cm}, 46.47$ $\mathrm{cm}$, and $16.59 \mathrm{~cm}$. The differences between province for udder measurements (except udder circumference) were significant $(\mathrm{P}<0.05)$. Teat length, teat diameter of the determined goats, as well as the distance between teats and distance from teat to the floor for the same month were found averagely as $7.94 \mathrm{~cm}, 2.98 \mathrm{~cm}, 14.35 \mathrm{~cm}$ and 37.30 $\mathrm{cm}$ for the first four flocks. On the other hand, these teat measurements were found respectively as $7.52 \mathrm{~cm}, 2.75$ $\mathrm{cm}, 14.95 \mathrm{~cm}$, and $37.04 \mathrm{~cm}$ for goats in the last four flocks. While there were significant differences for teat length and the distance between teats $(\mathrm{P}<0.05)$, nonsignificant differences were found for the other teat traits $(\mathrm{P}>0.05)$. The similar situation for teat traits was appeared in the second measurement period (April). Values regarding udder and teat (except for the distance from teat to the floor) which increased in April depending on the course of lactation began to relatively decrease as from May. For this measurement month and the other ones, The highest values were observed in the first flock whereas the lowest values were observed in the seventh flock. Generally, While the significant differences were found for udder depth and udder circumference both flocks in Antalya and the flocks in Burdur $(\mathrm{P}<0.05)$ provinces, the significant levels were detected as changeable for other traits. Among the aforementioned flocks; udder depth, udder circumference, udder width, teat length, teat diameter, the distance between teats, and distance from teat to the floor in June in the first flock were 
A
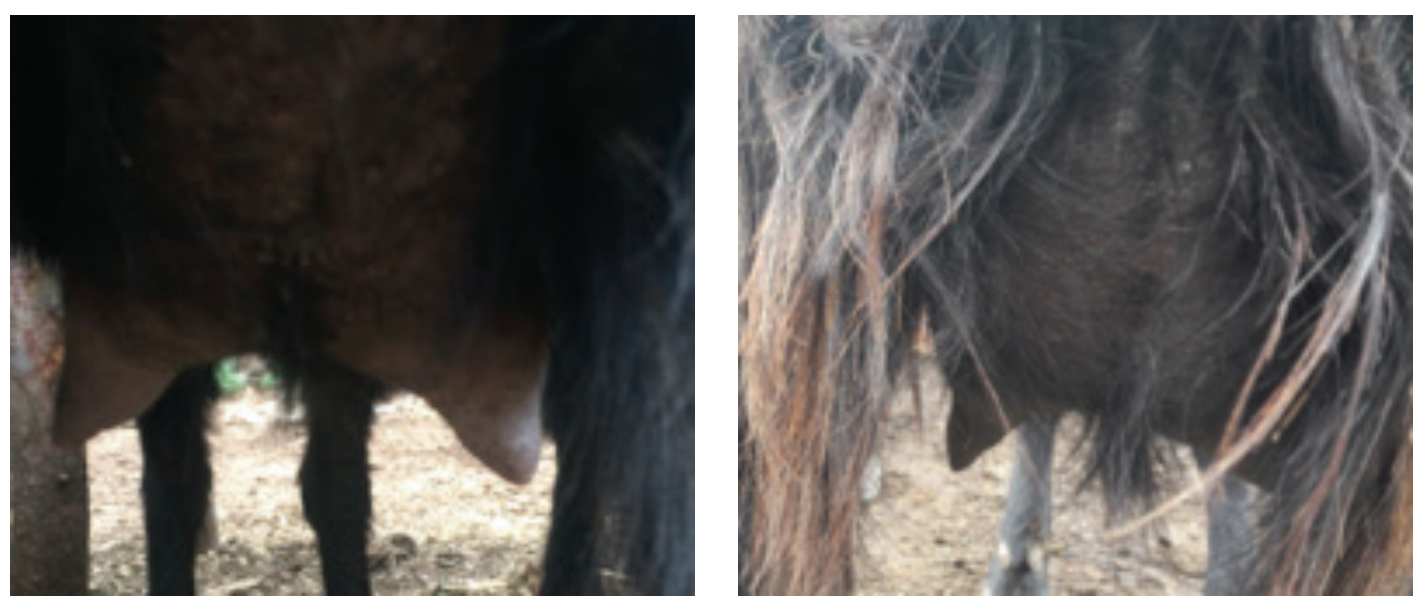

B
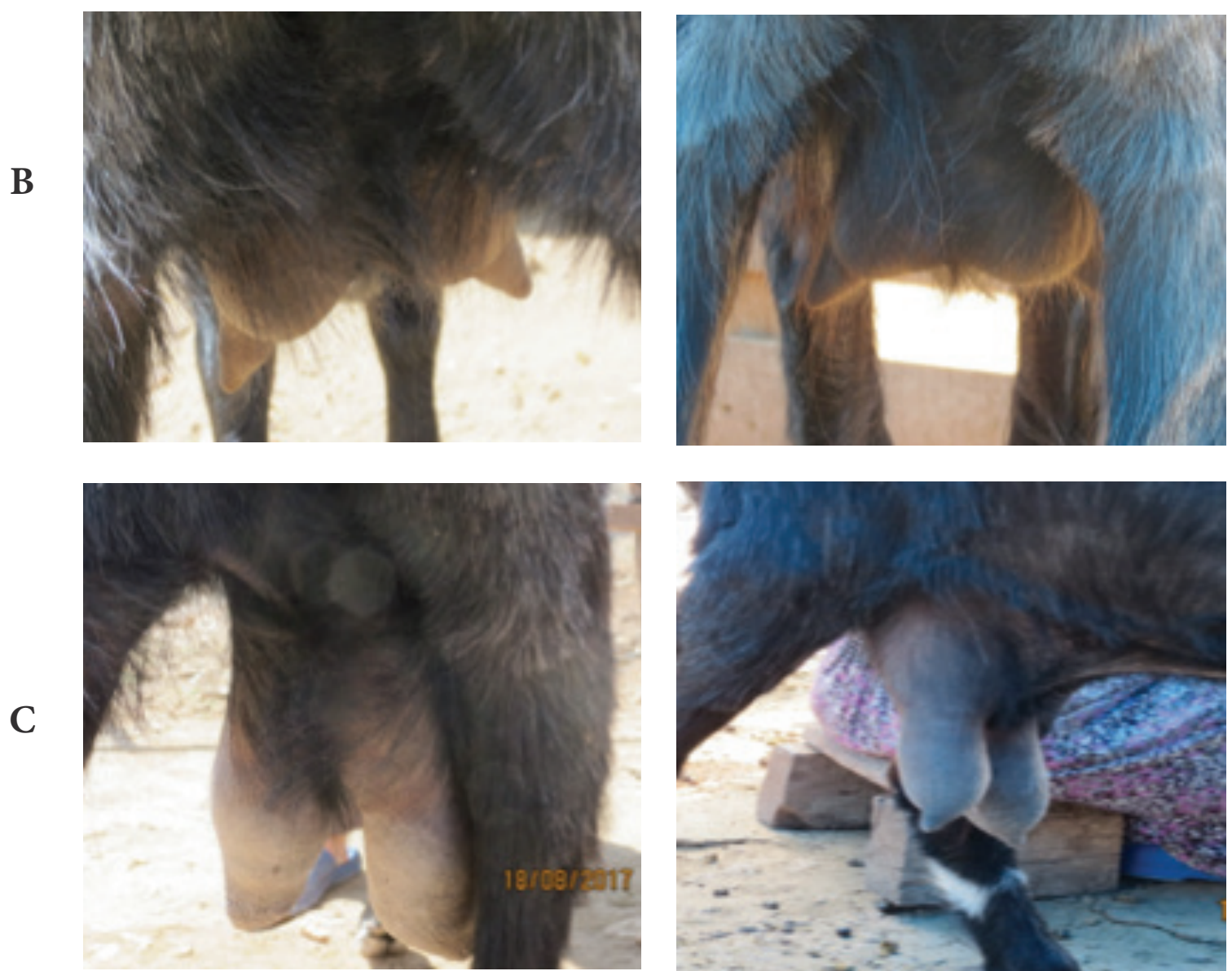

Figure 1. Udder types in Honamlı goats (A: Funnel; B: Sheep shape udder; C: Too divided)

\begin{tabular}{|l|c|c|c|c|c|}
\hline Flocks & $\begin{array}{l}\text { General } \\
\text { appearance }\end{array}$ & $\begin{array}{l}\text { Dairy } \\
\text { strength }\end{array}$ & $\begin{array}{l}\text { Body } \\
\text { capacity }\end{array}$ & $\begin{array}{l}\text { Mammary } \\
\text { system }\end{array}$ & $\begin{array}{l}\text { Total } \\
\text { Score }\end{array}$ \\
\hline Flock 1 & 27 & 16 & 7 & 28 & 78 \\
\hline Flock 2 & 26 & 16 & 7 & 26 & 75 \\
\hline Flock 3 & 27 & 16 & 6 & 27 & 76 \\
\hline Flock 4 & 25 & 15 & 7 & 27 & 74 \\
\hline Flock 5 & 26 & 15 & 6 & 27 & 74 \\
\hline Flock 6 & 26 & 15 & 6 & 26 & 73 \\
\hline Flock 7 & 26 & 14 & 6 & 26 & 72 \\
\hline Flock 8 & 26 & 16 & 7 & 26 & 75 \\
\hline
\end{tabular}

Table 1. Mean scores of goats according to ADGA scoring system 
Table 2. Udder and teat measurements of goats $($ March $)(\mathrm{cm})( \pm)$

\begin{tabular}{|c|c|c|c|c|c|c|c|}
\hline Flocks & Udder depth & $\begin{array}{l}\text { Udder } \\
\text { circumference }\end{array}$ & Udder width & Teat length & $\begin{array}{c}\text { Teat } \\
\text { diameter }\end{array}$ & $\begin{array}{c}\text { Distance between } \\
\text { teats }\end{array}$ & $\begin{array}{l}\text { Distance to floor } \\
\text { from the teat }\end{array}$ \\
\hline Flock 1 & $22.03^{\mathrm{a}} \pm 0.08$ & $47.11^{\mathrm{a}} \pm 0.13$ & $17.23^{\mathrm{a}} \pm 0.15$ & $8.15^{\mathrm{a}} \pm 0.30$ & $3.19 \pm 0.16$ & $14.03^{b} \pm 0.42$ & $37.70 \pm 0.43$ \\
\hline Flock 2 & $21.22^{b} \pm 0.18$ & $46.32^{b} \pm 0.18$ & $16.70^{b} \pm 0.33$ & $7.77^{\mathrm{b}} \pm 0.23$ & $2.80 \pm 0.27$ & $15.06^{\mathrm{a}} \pm 0.21$ & $37.14 \pm 0.30$ \\
\hline Flock 3 & $21.70^{\mathrm{ab}} \pm 0.23$ & $47.0^{\mathrm{a}} \pm 0.22$ & $17.04^{\mathrm{ab}} \pm 0.31$ & $8.02^{\mathrm{ab}} \pm 0.11$ & $3.06 \pm 0.39$ & $14.13^{\mathrm{b}} \pm 0.20$ & $37.13 \pm 0.24$ \\
\hline Flock 4 & $21.56^{\mathrm{b}} \pm 0.11$ & $46.75^{\mathrm{b}} \pm 0.34$ & $16.83^{\mathrm{b}} \pm 0.22$ & $7.8^{\mathrm{b}} \pm 0.27$ & $2.88 \pm 0.41$ & $14.20^{b_{ \pm}} 0.31$ & $37.25 \pm 0.36$ \\
\hline$P$ & $0.033^{*}$ & $0.028^{*}$ & $0.041^{*}$ & $0.037^{*}$ & $0.062^{\mathrm{ns}}$ & $0.039^{*}$ & $0.076^{\mathrm{ns}}$ \\
\hline Flock 5 & $21.72^{\mathrm{a}} \pm 0.09$ & $47.05^{\mathrm{a}} \pm 0.12$ & $16.77 \pm 0.18$ & $7.72^{\mathrm{a}} \pm 0.17$ & $2.84 \pm 0.19$ & $15.00 \pm 0.16$ & $37.33^{\mathrm{a}} \pm 0.22$ \\
\hline Flock 6 & $21.18^{\mathrm{b}} \pm 0.22$ & $46.28^{b} \pm 0.21$ & $16.60 \pm 0.13$ & $7.49^{b} \pm 0.23$ & $2.77 \pm 0.30$ & $14.89 \pm 0.31$ & $36.89^{b} \pm 0.27$ \\
\hline Flock 7 & $21.03^{b} \pm 0.07$ & $45.88^{c} \pm 0.23$ & $16.28 \pm 0.32$ & $7.36^{b} \pm 0.11$ & $2.68 \pm 0.27$ & $15.09 \pm 0.26$ & $36.70^{c} \pm 0.13$ \\
\hline Flock 8 & $21.66^{\mathrm{a}} \pm 0.24$ & $46.65^{\mathrm{ab}} \pm 0.10$ & $16.71 \pm 0.19$ & $7.55^{\mathrm{b}} \pm 0.14$ & $2.74 \pm 0.15$ & $14.80 \pm 0.31$ & $37.22^{\mathrm{ab}_{ \pm}} \pm 0.34$ \\
\hline$P$ & $0.047^{*}$ & $0.022^{*}$ & $0.055^{\mathrm{ns}}$ & $0.040^{*}$ & $0.179^{\text {ns }}$ & $0.201^{\mathrm{ns}}$ & $0.038^{*}$ \\
\hline $\begin{array}{l}\text { Mean of Antalya } \\
\text { (Flock 1-4) }\end{array}$ & $21.62 \pm 0.16$ & $46.80 \pm 0.23$ & $16.95 \pm 0.25$ & $7.94 \pm 0.23$ & $2.98 \pm 0.31$ & $14.35 \pm 0.28$ & $37.30 \pm 0.33$ \\
\hline $\begin{array}{l}\text { Mean of Burdur } \\
\text { (Flock 5-8) }\end{array}$ & $21.40 \pm 0.15$ & $46.47 \pm 0.17$ & $16.59 \pm 0.21$ & $7.52 \pm 0.17$ & $2.75 \pm 0.23$ & $14.95 \pm 0.25$ & $37.04 \pm 0.23$ \\
\hline $\mathrm{P}$ & $0.041^{*}$ & $0.075^{\text {ns }}$ & $0.036^{*}$ & $0.029 *$ & $0.243^{\mathrm{ns}}$ & $0.031^{*}$ & $0.066^{\mathrm{ns}}$ \\
\hline
\end{tabular}

Table 3. Udder and teat measurements of goats (April) $(\mathrm{cm})( \pm)$

\begin{tabular}{|c|c|c|c|c|c|c|c|}
\hline Flocks & Udder depth & $\begin{array}{l}\text { Udder } \\
\text { circumference }\end{array}$ & $\begin{array}{l}\text { Udder } \\
\text { width }\end{array}$ & Teat length & $\begin{array}{c}\text { Teat } \\
\text { diameter }\end{array}$ & $\begin{array}{c}\text { Distance } \\
\text { between teats }\end{array}$ & $\begin{array}{l}\text { Distance to floor } \\
\text { from the teat }\end{array}$ \\
\hline Flock 1 & $22.18^{\mathrm{a}} \pm 0.12$ & $47.30^{\mathrm{a}} \pm 0.19$ & $17.35^{\mathrm{a}} \pm 0.22$ & $8.26^{\mathrm{a}} \pm 0.15$ & $3.30 \pm 0.21$ & $14.15^{b} \pm 0.14$ & $37.58 \pm 0.26$ \\
\hline Flock 2 & $21.36^{\mathrm{b}} \pm 0.26$ & $46.51^{c} \pm 0.24$ & $16.83^{b} \pm 0.16$ & $7.89^{b} \pm 0.12$ & $2.91 \pm 0.30$ & $15.18^{a} \pm 0.10$ & $37.08 \pm 0.15$ \\
\hline Flock 3 & $21.81^{\mathrm{ab}} \pm 0.30$ & $47.19^{\mathrm{ab}} \pm 0.15$ & $17.14^{a} \pm 0.28$ & $8.14^{a b} \pm 0.20$ & $3.14 \pm 0.18$ & $14.22^{\mathrm{b}} \pm 0.07$ & $37.04 \pm 0.21$ \\
\hline Flock 4 & $21.58^{\mathrm{b}} \pm 0.16$ & $46.88^{\mathrm{b}} \pm 0.43$ & $16.92^{\mathrm{b}} \pm 0.19$ & $7.95^{b} \pm 0.18$ & $2.97 \pm 0.34$ & $14.34^{\mathrm{b}} \pm 0.19$ & $37.19 \pm 0.13$ \\
\hline $\mathrm{P}$ & $0.039^{*}$ & $0.020 *$ & $0.037^{*}$ & $0.041^{*}$ & $0.053^{\text {ns }}$ & $0.045^{*}$ & $0.060^{\text {ns }}$ \\
\hline Flock 5 & $21.90^{\mathrm{a}} \pm 0.23$ & $47.17^{\mathrm{a}} \pm 0.08$ & $16.89 \pm 0.20$ & $7.84^{\mathrm{a}} \pm 0.12$ & $2.94 \pm 0.20$ & $15.07 \pm 0.14$ & $37.24^{\mathrm{a}} \pm 0.16$ \\
\hline Flock 6 & $21.27^{b} \pm 0.11$ & $46.40^{\mathrm{b}} \pm 0.09$ & $16.76 \pm 0.16$ & $7.64^{b} \pm 0.14$ & $2.91 \pm 0.19$ & $14.97 \pm 0.30$ & $36.76^{\mathrm{b}} \pm 0.17$ \\
\hline Flock 7 & $21.11^{b} \pm 0.14$ & $45.9^{c} \pm 0.18$ & $16.41 \pm 0.23$ & $7.52^{b} \pm 0.09$ & $2.80 \pm 0.14$ & $15.18 \pm 0.18$ & $36.58^{b} \pm 0.23$ \\
\hline Flock 8 & $21.79^{a} \pm 0.13$ & $46.76^{\mathrm{ab}} \pm 0.07$ & $16.83 \pm 0.17$ & $7.66^{\mathrm{b}} \pm 0.23$ & $2.89 \pm 0.12$ & $14.93 \pm 0.22$ & $37.16^{\mathrm{a}} \pm 0.21$ \\
\hline $\mathrm{P}$ & $0.035^{*}$ & $0.018^{*}$ & $0.142^{\text {ns }}$ & $0.047^{*}$ & $0.327^{\mathrm{ns}}$ & $0.470^{\mathrm{ns}}$ & $0.043 *$ \\
\hline $\begin{array}{l}\text { Mean of Antalya } \\
\text { (Flock 1-4) }\end{array}$ & $21.73 \pm 0.21$ & $46.97 \pm 0.22$ & $17.06 \pm 0.23$ & $8.05 \pm 0.17$ & $3.08 \pm 0.26$ & $14.47 \pm 0.12$ & $37.22 \pm 0.18$ \\
\hline $\begin{array}{l}\text { Mean of Burdur } \\
\text { (Flock 5-8) }\end{array}$ & $21.51 \pm 0.17$ & $46.57 \pm 0.11$ & $16.72 \pm 0.19$ & $7.67 \pm 0.15$ & $2.88 \pm 0.17$ & $15.04 \pm 0.20$ & $36.94 \pm 0.19$ \\
\hline $\mathrm{P}$ & $0.038^{*}$ & $0.045^{*}$ & $0.057^{\mathrm{ns}}$ & $0.035^{*}$ & $0.189^{\text {ns }}$ & $0.024^{*}$ & $0.203^{\mathrm{ns}}$ \\
\hline
\end{tabular}


Table 4. Udder and teat measurements of goats (May) $(\mathrm{cm})( \pm)$

\begin{tabular}{|c|c|c|c|c|c|c|c|}
\hline Flocks & Udder depth & $\begin{array}{l}\text { Udder } \\
\text { circumference }\end{array}$ & Udder width & Teat length & $\begin{array}{c}\text { Teat } \\
\text { diameter }\end{array}$ & $\begin{array}{c}\text { Distance } \\
\text { between teats }\end{array}$ & $\begin{array}{l}\text { Distance to floor } \\
\text { from the teat }\end{array}$ \\
\hline Flock 1 & $21.88^{\mathrm{a}} \pm 0.12$ & $47.02^{\mathrm{a}} \pm 0.19$ & $17.06^{\mathrm{a}} \pm 0.22$ & $8.06^{\mathrm{a}} \pm 0.15$ & $3.03 \pm 0.21$ & $13.95^{\mathrm{b}} \pm 0.14$ & $37.79 \pm 0.26$ \\
\hline Flock 2 & $21.01^{\mathrm{b}} \pm 0.26$ & $46.18^{c} \pm 0.24$ & $16.52^{\mathrm{b}} \pm 0.19$ & $7.66^{c} \pm 0.12$ & $2.67 \pm 0.30$ & $14.86^{\mathrm{a}} \pm 0.10$ & $37.18 \pm 0.15$ \\
\hline Flock 3 & $21.49^{\mathrm{a}} \pm 0.30$ & $46.89^{a} \pm 0.15$ & $16.76^{\mathrm{b}} \pm 0.28$ & $7.92^{\mathrm{ab}} \pm 0.20$ & $2.94 \pm 0.18$ & $14.08^{b} \pm 0.07$ & $37.19 \pm 0.21$ \\
\hline Flock 4 & $21.40^{\mathrm{ab}} \pm 0.16$ & $46.58^{\mathrm{ab}} \pm 0.43$ & $16.64^{b} \pm 0.17$ & $7.76^{\mathrm{bc}} \pm 0.18$ & $2.81 \pm 0.34$ & $14.16^{b} \pm 0.19$ & $37.33 \pm 0.13$ \\
\hline $\mathrm{P}$ & $0.027^{*}$ & $0.020^{*}$ & $0.031^{*}$ & $0.018^{*}$ & $0.052^{\mathrm{ns}}$ & $0.019^{*}$ & $0.106^{\mathrm{ns}}$ \\
\hline Flock 5 & $21.58^{\mathrm{a}} \pm 0.23$ & $46.92^{\mathrm{a}} \pm 0.08$ & $16.62 \pm 0.20$ & $7.63^{\mathrm{a}} \pm 0.12$ & $2.77 \pm 0.20$ & $14.91 \pm 0.14$ & $37.44 \pm 0.16$ \\
\hline Flock 6 & $21.10^{b_{ \pm}} 0.11$ & $46.17^{b c} \pm 0.09$ & $16.48 \pm 0.16$ & $7.35^{b} \pm 0.14$ & $2.64 \pm 0.19$ & $14.77 \pm 0.30$ & $36.96 \pm 0.17$ \\
\hline Flock 7 & $20.89^{b} \pm 0.14$ & $45.73^{c} \pm 0.18$ & $16.19 \pm 0.23$ & $7.27^{b} \pm 0.09$ & $2.55 \pm 0.14$ & $15.01 \pm 0.18$ & $36.85 \pm 0.23$ \\
\hline Flock 8 & $21.55^{\mathrm{a}} \pm 0.13$ & $46.54^{\mathrm{ab}} \pm 0.07$ & $16.58 \pm 0.17$ & $7.48^{\mathrm{b}} \pm 0.23$ & $2.66 \pm 0.12$ & $14.69 \pm 0.22$ & $37.36 \pm 0.21$ \\
\hline$P$ & $0.040^{*}$ & $0.037^{*}$ & $0.104^{\mathrm{ns}}$ & $0.032^{*}$ & $0.298^{\mathrm{ns}}$ & $0.057^{\mathrm{ns}}$ & $0.097^{\mathrm{ns}}$ \\
\hline $\begin{array}{l}\text { Mean of Antalya } \\
\text { (Flock 1-4) }\end{array}$ & $21.45 \pm 0.21$ & $46.67 \pm 0.22$ & $16.74 \pm 0.22$ & $7.85 \pm 0.17$ & $2.86 \pm 0.26$ & $14.26 \pm 0.12$ & $37.36 \pm 0.18$ \\
\hline $\begin{array}{l}\text { Mean of Burdur } \\
\text { (Flock 5-8) }\end{array}$ & $21.28 \pm 0.17$ & $46.33 \pm 0.11$ & $16.45 \pm 0.19$ & $7.43 \pm 0.15$ & $2.65 \pm 0.17$ & $14.84 \pm 0.20$ & $37.14 \pm 0.19$ \\
\hline$P$ & $0.052^{\mathrm{ns}}$ & $0.109^{\text {ns }}$ & $0.061^{\mathrm{ns}}$ & $0.043^{*}$ & $0.164^{\mathrm{ns}}$ & $0.023^{*}$ & $0.074^{\mathrm{ns}}$ \\
\hline
\end{tabular}

Table 5. Udder and teat measurements of goats (June) $(\mathrm{cm})( \pm)$

\begin{tabular}{|c|c|c|c|c|c|c|c|}
\hline Flocks & Udder depth & $\begin{array}{l}\text { Udder } \\
\text { circumference }\end{array}$ & Udder width & Teat length & $\begin{array}{c}\text { Teat } \\
\text { diameter }\end{array}$ & $\begin{array}{c}\text { Distance } \\
\text { between teats }\end{array}$ & $\begin{array}{l}\text { Distance to floor } \\
\text { from the teat }\end{array}$ \\
\hline Flock 1 & $21.12^{\mathrm{a}} \pm 0.18$ & $46.33^{a} \pm 0.26$ & $16.51^{\mathrm{a}} \pm 0.23$ & $7.95^{\mathrm{a}} \pm 0.11$ & $2.88 \pm 0.10$ & $13.77^{b} \pm 0.12$ & $37.97^{\mathrm{a}} \pm 0.21$ \\
\hline Flock 2 & $20.29^{c} \pm 0.25$ & $45.52^{c} \pm 0.22$ & $15.88^{\mathrm{b}} \pm 0.11$ & $7.34^{b} \pm 0.23$ & $2.52 \pm 0.20$ & $14.66^{\mathrm{a}} \pm 0.17$ & $37.42^{b} \pm 0.08$ \\
\hline Flock 3 & $20.68^{b} \pm 0.15$ & $46.09^{\mathrm{ab}} \pm 0.11$ & $16.16^{\mathrm{b}} \pm 0.24$ & $7.55^{\mathrm{ab}} \pm 0.18$ & $2.77 \pm 0.15$ & $13.89^{\mathrm{b}} \pm 0.10$ & $37.44^{b} \pm 0.27$ \\
\hline Flock 4 & $20.71^{b} \pm 0.17$ & $45.89^{b} \pm 0.13$ & $16.02^{\mathrm{b}} \pm 0.15$ & $7.49^{b} \pm 0.22$ & $2.60 \pm 0.31$ & $13.95^{\mathrm{b}} \pm 0.16$ & $37.68^{\mathrm{a}} \pm 0.19$ \\
\hline$P$ & $0.028^{*}$ & $0.034 *$ & $0.040^{*}$ & 0.044* & $0.201^{\mathrm{ns}}$ & $0.033^{*}$ & $0.046^{*}$ \\
\hline Flock 5 & $20.78^{\mathrm{ab}} \pm 0.11$ & $46.15^{\mathrm{a}} \pm 0.23$ & $16.01 \pm 0.21$ & $7.37 \pm 0.15$ & $2.56 \pm 0.23$ & $14.75 \pm 0.21$ & $37.78^{a} \pm 0.30$ \\
\hline Flock 6 & $20.39^{b c} \pm 0.20$ & $45.38^{b} \pm 0.17$ & $15.89 \pm 0.20$ & $7.14 \pm 0.11$ & $2.49 \pm 0.14$ & $14.58 \pm 0.17$ & $37.21^{\mathrm{b}} \pm 0.22$ \\
\hline Flock 7 & $20.15^{c} \pm 0.18$ & $45.02^{\mathrm{b}} \pm 0.18$ & $15.42 \pm 0.24$ & $7.08 \pm 0.20$ & $2.37 \pm 0.07$ & $14.81 \pm 0.23$ & $36.95^{\mathrm{b}} \pm 0.13$ \\
\hline Flock 8 & $20.88^{\mathrm{a}} \pm 0.21$ & $45.91^{\mathrm{b}} \pm 0.12$ & $15.96 \pm 0.18$ & $7.33 \pm 0.13$ & $2.48 \pm 0.21$ & $14.48 \pm 0.29$ & $37.84^{\mathrm{a}} \pm 0.10$ \\
\hline$P$ & $0.011^{*}$ & $0.041^{*}$ & $0.071^{\mathrm{ns}}$ & $0.208^{\mathrm{ns}}$ & $0.315^{\mathrm{ns}}$ & $0.169^{\text {ns }}$ & $0.030^{*}$ \\
\hline $\begin{array}{l}\text { Mean of Antalya } \\
\text { (Flock 1-4) }\end{array}$ & $20.70 \pm 0.19$ & $45.95 \pm 0.18$ & $16.14 \pm 0.17$ & $7.58 \pm 0.19$ & $2.69 \pm 0.21$ & $14.06 \pm 0.13$ & $37.62 \pm 0.18$ \\
\hline $\begin{array}{l}\text { Mean of Burdur } \\
\text { (Flock 5-8) }\end{array}$ & $20.56 \pm 0.17$ & $45.61 \pm 0.17$ & $15.82 \pm 0.21$ & $7.22 \pm 0.14$ & $2.47 \pm 0.16$ & $14.65 \pm 0.22$ & $37.44 \pm 0.21$ \\
\hline$P$ & $0.089^{\text {ns }}$ & $0.056^{\text {ns }}$ & $0.047^{*}$ & $0.024 *$ & $0.121^{\mathrm{ns}}$ & $0.018^{*}$ & $0.192^{\mathrm{ns}}$ \\
\hline
\end{tabular}


Table 6. Udder and teat measurements of goats (July) $(\mathrm{cm})( \pm)$

\begin{tabular}{|c|c|c|c|c|c|c|c|}
\hline Flocks & Udder depth & $\begin{array}{l}\text { Udder } \\
\text { circumference }\end{array}$ & Udder width & Teat length & $\begin{array}{c}\text { Teat } \\
\text { diameter }\end{array}$ & $\begin{array}{c}\text { Distance } \\
\text { between teats }\end{array}$ & $\begin{array}{l}\text { Distance to floor } \\
\text { from the teat }\end{array}$ \\
\hline Flock 1 & $20.61^{\mathrm{a}} \pm 0.10$ & $45.88^{\mathrm{a}} \pm 0.24$ & $16.07^{a} \pm 0.26$ & $7.83^{\mathrm{a}} \pm 0.20$ & $2.67 \pm 0.08$ & $13.61^{b} \pm 0.07$ & $38.25 \pm 0.19$ \\
\hline Flock 2 & $19.82^{b} \pm 0.21$ & $45.04^{b} \pm 0.23$ & $15.34^{c} \pm 0.09$ & $7.20^{\mathrm{b}} \pm 0.16$ & $2.33 \pm 0.17$ & $14.51^{\mathrm{a}} \pm 0.19$ & $37.75 \pm 0.16$ \\
\hline Flock 3 & $20.16^{\mathrm{b}} \pm 0.13$ & $45.52^{\mathrm{a}} \pm 0.17$ & $15.77^{a b} \pm 0.16$ & $7.39^{b} \pm 0.12$ & $2.53 \pm 0.22$ & $13.77^{b} \pm 0.14$ & $37.88 \pm 0.23$ \\
\hline Flock 4 & $20.18^{\mathrm{b}} \pm 0.12$ & $45.28^{\mathrm{b}} \pm 0.19$ & $15.64^{b} \pm 0.14$ & $7.23^{b} \pm 0.21$ & $2.38 \pm 0.30$ & $13.81^{\mathrm{b}} \pm 0.22$ & $37.93 \pm 0.14$ \\
\hline$P$ & $0.034 *$ & $0.026^{*}$ & $0.042 *$ & $0.021^{*}$ & $0.094^{\mathrm{ns}}$ & $0.022 *$ & $0.302^{\text {ns }}$ \\
\hline Flock 5 & $20.26^{\mathrm{b}} \pm 0.17$ & $45.70^{\mathrm{a}} \pm 0.25$ & $15.66^{\mathrm{a}} \pm 0.19$ & $7.23 \pm 0.11$ & $2.32 \pm 0.29$ & $14.60 \pm 0.28$ & $38.08^{\mathrm{a}} \pm 0.16$ \\
\hline Flock 6 & $19.95^{\mathrm{b}} \pm 0.21$ & $44.87^{b} \pm 0.20$ & $15.37^{\mathrm{ab}} \pm 0.18$ & $7.01 \pm 0.16$ & $2.23 \pm 0.16$ & $14.40 \pm 0.14$ & $38.02^{\mathrm{a}} \pm 0.23$ \\
\hline Flock 7 & $20.63^{a} \pm 0.14$ & $44.53^{b} \pm 0.17$ & $14.96^{\mathrm{b}} \pm 0.20$ & $6.91 \pm 0.23$ & $2.19 \pm 0.12$ & $14.63 \pm 0.25$ & $37.13^{b} \pm 0.14$ \\
\hline Flock 8 & $20.34^{b} \pm 0.19$ & $45.52^{\mathrm{a}} \pm 0.13$ & $15.74^{\mathrm{a}} \pm 0.17$ & $7.22 \pm 0.08$ & $2.27 \pm 0.16$ & $14.36 \pm 0.20$ & $37.99^{a} \pm 0.11$ \\
\hline$P$ & $0.028 *$ & $0.042 *$ & $0.033^{*}$ & $0.088^{\text {ns }}$ & $0.203^{\mathrm{ns}}$ & $0.182^{\mathrm{ns}}$ & $0.035^{*}$ \\
\hline $\begin{array}{l}\text { Mean of Antalya } \\
\text { (Flock 1-4) }\end{array}$ & $20.19 \pm 0.15$ & $45.43 \pm 0.21$ & $15.70 \pm 0.15$ & $7.41 \pm 0.20$ & $2.46 \pm 0.19$ & $13.92 \pm 0.15$ & $37.95 \pm 0.18$ \\
\hline $\begin{array}{l}\text { Mean of Burdur } \\
\text { (Flock 5-8) }\end{array}$ & $20.29 \pm 0.18$ & $45.15 \pm 0.19$ & $15.43 \pm 0.18$ & $7.09 \pm 0.15$ & $2.27 \pm 0.18$ & $14.50 \pm 0.22$ & $37.80 \pm 0.17$ \\
\hline$P$ & $0.244^{\mathrm{ns}}$ & $0.070^{\mathrm{ns}}$ & $0.052^{\mathrm{ns}}$ & $0.037^{*}$ & $0.069^{\text {ns }}$ & $0.016^{*}$ & $0.117^{\text {ns }}$ \\
\hline
\end{tabular}

Table 7. Udder and teat measurements of goats (August) $(\mathrm{cm})( \pm$ )

\begin{tabular}{|c|c|c|c|c|c|c|c|}
\hline Flocks & Udder depth & $\begin{array}{l}\text { Udder } \\
\text { circumference }\end{array}$ & Udder width & Teat length & $\begin{array}{c}\text { Teat } \\
\text { diameter }\end{array}$ & $\begin{array}{c}\text { Distance } \\
\text { between teats }\end{array}$ & $\begin{array}{c}\text { Distance to } \\
\text { floor from } \\
\text { the teat }\end{array}$ \\
\hline Flock 1 & $20.02^{\mathrm{a}} \pm 0.20$ & $45.22^{a} \pm 0.20$ & $15.61^{a} \pm 0.21$ & $7.65^{\mathrm{a}} \pm 0.10$ & $2.44 \pm 0.13$ & $13.40^{\mathrm{b}} \pm 0.14$ & $38.53 \pm 0.12$ \\
\hline Flock 2 & $19.29^{b} \pm 0.18$ & $44.69^{b} \pm 0.21$ & $14.72^{b c} \pm 0.11$ & $7.02^{b} \pm 0.13$ & $2.12 \pm 0.16$ & $14.28^{\mathrm{a}} \pm 0.16$ & $38.11 \pm 0.23$ \\
\hline Flock 3 & $19.71^{\mathrm{a}} \pm 0.10$ & $45.03^{\mathrm{ab}} \pm 0.14$ & $15.31^{\mathrm{a}} \pm 0.23$ & $7.17^{b} \pm 0.15$ & $2.29 \pm 0.20$ & $13.53^{b} \pm 0.09$ & $38.04 \pm 0.14$ \\
\hline Flock 4 & $19.72^{a} \pm 0.07$ & $44.79^{b} \pm 0.31$ & $15.16^{\mathrm{ab}} \pm 0.17$ & $7.04^{b} \pm 0.11$ & $2.21 \pm 0.28$ & $13.74^{\mathrm{a}} \pm 0.20$ & $38.24 \pm 0.17$ \\
\hline $\mathrm{P}$ & $0.031 *$ & $0.040^{*}$ & $0.026^{*}$ & $0.044^{*}$ & $0.070^{\text {ns }}$ & $0.035^{*}$ & $0.062^{\text {ns }}$ \\
\hline Flock 5 & $19.73^{a} \pm 0.15$ & $45.13^{a} \pm 0.15$ & $15.09^{\mathrm{ab}} \pm 0.11$ & $7.07 \pm 0.19$ & $2.14 \pm 0.22$ & $14.48 \pm 0.28$ & $38.51 \mathrm{a} \pm 0.21$ \\
\hline Flock 6 & $19.38^{\mathrm{b}} \pm 0.17$ & $44.31^{b_{ \pm}} 0.23$ & $14.81^{b_{ \pm}} 0.14$ & $6.88 \pm 0.12$ & $2.09 \pm 0.18$ & $14.27 \pm 0.20$ & $38.44^{\mathrm{a}} \pm 0.17$ \\
\hline Flock 7 & $20.07^{a} \pm 0.10$ & $44.02^{b} \pm 0.19$ & $14.35^{\mathrm{c}} \pm 0.21$ & $6.70 \pm 0.26$ & $2.04 \pm 0.11$ & $14.51 \pm 0.29$ & $37.54^{b} \pm 0.08$ \\
\hline Flock 8 & $19.86^{a} \pm 0.14$ & $45.03^{a} \pm 0.14$ & $15.22^{\mathrm{a}} \pm 0.27$ & $7.01 \pm 0.33$ & $2.09 \pm 0.10$ & $14.17 \pm 0.24$ & $38.40^{\mathrm{a}} \pm 0.11$ \\
\hline$P$ & $0.027^{*}$ & $0.038^{*}$ & $0.017 *$ & $0.090^{\text {ns }}$ & $0.123^{\text {ns }}$ & $0.201^{\mathrm{ns}}$ & $0.018^{*}$ \\
\hline $\begin{array}{l}\text { Mean of Antalya } \\
\text { (Flock 1-4) }\end{array}$ & $19.68 \pm 0.14$ & $44.95 \pm 0.22$ & $15.11 \pm 0.19$ & $7.22 \pm 0.13$ & $2.26 \pm 0.17$ & $13.72 \pm 0.15$ & $38.22 \pm 0.16$ \\
\hline $\begin{array}{l}\text { Mean of Burdur } \\
\text { (Flock 5-8) }\end{array}$ & $19.76 \pm 0.13$ & $44.61 \pm 0.18$ & $14.87 \pm 0.19$ & $6.91 \pm 0.22$ & $2.10 \pm 0.16$ & $14.35 \pm 0.22$ & $38.20 \pm 0.14$ \\
\hline $\mathrm{P}$ & $0.214^{\mathrm{ns}}$ & $0.059^{\text {ns }}$ & $0.122^{\mathrm{ns}}$ & $0.110^{\text {ns }}$ & $0.075^{\mathrm{ns}}$ & $0.037 *$ & $0.544^{\mathrm{ns}}$ \\
\hline
\end{tabular}


determined respectively as $21.12 \mathrm{~cm}, 46.33 \mathrm{~cm}, 16.51 \mathrm{~cm}$, $7.95 \mathrm{~cm}, 2.88 \mathrm{~cm}, 13.77 \mathrm{~cm}$ and $37.97 \mathrm{~cm}$. The same udder and teat measurements were respectively $20.02 \mathrm{~cm}, 45.22$ $\mathrm{cm}, 15.61 \mathrm{~cm}, 7.65 \mathrm{~cm}, 2.44 \mathrm{~cm}, 13.40 \mathrm{~cm}$ and $38.53 \mathrm{~cm}$ in the last measurement month (August). August values of the lowest flock (seventh flock) were lower than other flocks and were determined respectively as $20.07 \mathrm{~cm}, 44.02$ $\mathrm{cm}, 14.35 \mathrm{~cm}, 6.70 \mathrm{~cm}, 2.04 \mathrm{~cm}, 14.51 \mathrm{~cm}$, and $37.54 \mathrm{~cm}$. With regards to the udder and teat traits (except distance between teats), goats in the province of Antalya had higher values than goats in the province of Burdur in all observation periods. While the differences between provinces for udder traits (depth, circumference and width) were not statistically significant in the last three measurement period, the same situation was said for the distance between teats and the distance to floor from the teat traits.

\section{Discussion ve Conclusion}

Number of studies aiming to reveal the dairy characteristics of native goats in Turkey is limited. There is a gradual increase in the number of enterprises rearing dairy goats in Teke Region which has a particular importance in terms of goat farming and these enterprises which are established with high costs also inspire traditional breeders causing them to want to improve dairy characteristics in their flocks, as well. In this respect, it is important to determine and reveal goats with a higher capability of dairying among Honamlı goats which are reared by the locals. From this point of view, it is seen that mean scores of Honamli goats determined for "general appearance, dairy characteristics, body capacity and udder structure" within the scope of the study are in the interval of acceptable values (70-79) in terms of dairy characteristics. In addition, as is mentioned above; the need for more detailed studies on this subject should be taken into consideration.

It is reported that both convenience for machine milking and udder type are important for higher milk yield in dairy goat farming and it will be possible to make a selection regarding high milk yield, increase of mastitis resistance and convenience for machine milking in case of determining type characteristics which show a high correlation with milk yield. ${ }^{16}$ As machine milking is not common among goats and sheep in Turkey, selection according to udder forms and related studies are not adequately addressed. ${ }^{17}$ Some udder and teat measurements of 40 Honamli goats determined within the scope of the study were specified with monthly measurements in a period of 6 months. In parallel with the genotype in the study; Elmaz et al. ${ }^{18}$ determined that the udder width, udder depth, udder circumference, teat length, teat width, the distance between teats and distance from teat to the ground in Honamli goats were $14.6 \mathrm{~cm}, 18.8 \mathrm{~cm}, 46.3 \mathrm{~cm}, 5.2 \mathrm{~cm}, 2.2 \mathrm{~cm}, 15.1 \mathrm{~cm}$, and $35.7 \mathrm{~cm}$, respectively. Starting from the results in the study; these values were observed to be higher than the aforementioned values, except for the last two characteristics, in general.

In the study investigating some yield characteristics of hair goats which constitute a large part of the goat population in Turkey; udder depth, udder circumference, teat length and teat diameter were measured respectively as $16.8 \mathrm{~cm}, 41.2$ $\mathrm{cm}, 2.4 \mathrm{~cm}$, and $1.7 \mathrm{~cm}$ throughout lactation. Similarly, in the study conducted by Atay et al. ${ }^{19}$ with 67 hair goats; teat diameter, teat length, distance between teats, udder depth, udder height and udder circumference were found respectively as $3.37 \mathrm{~cm}, 5.79 \mathrm{~cm}, 7.48 \mathrm{~cm}, 18.66 \mathrm{~cm}, 34.96 \mathrm{~cm}$, and $40.75 \mathrm{~cm}$. In the study conducted by Erol et al. ${ }^{20}$ with Ankara goats, whose number is gradually decreasing and which are among important local gene resources, in the herds which were protected as a gene resource by the institute and breeders; it was determined that udder width, depth and circumference were measured respectively as $11.72 \mathrm{~cm}, 13.63 \mathrm{~cm}$, and $39.75 \mathrm{~cm}$.

In a study that was conducted with Turkish Saanen goats, which are among genotypes obtained by hybridizing local breeds and the Saanen goats which were brought to Turkey for developing the dairy goat farming and in this context forming especially dairy types by using culture breeds and are commonly reared in Turkey, un breeder conditions; udder width and udder circumference were found respectively as $10.45 \mathrm{~cm}$ and $34.46 \mathrm{~cm}$. In Akkeçi goats obtained by hybridizing the Kilis goats that are among important native dairy goats in Turkey with the Saanen breed; Cedden ${ }^{21}$ determined udder circumference as $38.73 \mathrm{~cm}, 37 \mathrm{~cm}$ and $44.20 \mathrm{~cm}$, left teat length as $3.77 \mathrm{~cm}, 4 \mathrm{~cm}$, and 4.50 $\mathrm{cm}$, right teat length as $3.6 \mathrm{~cm}, 3.50 \mathrm{~cm}$, and $4.40 \mathrm{~cm}$, and udder depth $11.55 \mathrm{~cm}, 11.50 \mathrm{~cm}$, and $13 \mathrm{~cm}$ respectively among udder measurements during the first, second and third lactation. In another study ${ }^{17}$ investigating the udder characteristics of Akkeçi; udder depth, udder circumference, teat diameter and teat length were found respectively as $13.97 \mathrm{~cm}, 47.32 \mathrm{~cm}, 2.22 \mathrm{~cm}$, and $4.40 \mathrm{~cm}$.

In order to conduct the activity of goat farming successfully, it is important to consider the aspect and way of breeding based on geographical and economic conditions of the region in question and also determine appropriate breeds. Breeders also demand for animals with a higher growth rate and a higher milk yield and it is seen that no selection has been made in the field conditions in terms of dairy characteristics. In this study, one of the characteristics of Honamlı goats was also examined in detail and thus, focal points of future comprehensive studies were determined in terms of these characteristics. It is thought that the study 
results are important in terms of identifying possible dairy varieties of Honamlı goats and sharing them with sector stakeholders.

\section{Acknowledgments}

This study has been supported by Scientific Research Studys Coordinator of Mehmet Akif Ersoy University (Study No: 0350-NAP-16).

\section{References}

1. Koyuncu M, Taşkın T, Kaymakçı M. Importance of goat milk in human health. Paper presented at: National Goat Congress; June 24-26, 2010; Çanakkale-Turkey.

2. Koyuncu M. Goat breeding strategy in the World and Turkey. Paper presented at: National Congress of Dairy Goat; May 26-27, 2005; İzmir-Turkey.

3. Gok B, Aktas AH, Dursun S. Honamli goat: the rising star of the Taurus mountains. Paper presented at: RBI 8th Global Conference on the Conservation of Animal Genetic Resources; October 4-8, 2011; Tekirdağ-Turkey.

4. Official Gazette of Turkish Republic. Communication on Supporting Principles of Animal Husbandry Practices, No: 2015/43, No: 29535, Ankara Basbakanlik Basimevi, Turkey; 2015.

5. Solaiman SG, ed. Goat Science and Production. 1sted. Iowa, Blackwell Publishing; 2010.

6. Gall C. Relationship between body conformation and production in dairy goats. J Dairy Sci. 1980;63:17681781 .

7. Le Du J, Benmederbel B. Machine milkability of Saanen breed, relationships with teat physical traits. Ann Zootech. 1984;33:375-384.

8. Alızadehasl M. Some important morphological traits in Kilis, Norduz and Honamlı indigenous goats breeds. Master thesis, Ankara University Health Science Institute, Ankara, 2011.

9. Karadag O, Soysal MI. The determination of some reproduction, growth and morphological traits in Honamlı goats breeds. Journal of Tekirdag Agricultural Faculty. 2018;15(1):135-142.

10. Manfredi E, Piacere A, Lahaye P, et al. Genetic parameters of type appraisal in Saanen and Alpine goats. Livest Prod Sci. 2001;70:183-189.

11. Janssens S, Vandepitte W. Genetic parameters for body measurements and linear type traits in Belgian Bleu du Maine, Suffolk and Texel sheep. Small Rumin Res. 2004;54:13-24.

12. ADGA. The American Dairy Goat Association Guide Book. 2014.
13. Thompson JR, Lee KL, Freeman AE. Evaluation of a linear type appraisal system for Holstein cattle. J Dairy Sci. 1983;66:325-331.

14. Unal N, Akcapinar H, Atasoy F, et al. Some udder traits and growth of lambs and phenotypic correlations between those of traits with milking traits and milk production measured by various milk estimation methods in Bafra sheep. Ankara Üniv Vet Fak Derg. 2008;55:117-124.

15. Minitab. Windows User's Guide. Version 16.1.1. Minitab Inc, State College, PA, USA, 2010.

16. Aktas ZM, Kaygisız A, Bas S. The relationships between Turkish Saanen goats milk yield traits, some udder measurements and SCC at Kahramanmaraş breeders conditions. KSU J Nat Sci. 2012;15(4):7-17.

17. Kor A, Keskin S, Karaca S, et al. The effect of age and lactation order on some udder measurements in Akkeçi. YYU J Agr Sci. 2004;14(2):105-111.

18. Elmaz O, Ozgel O, Agaoğlu AR, et al. Determination of reproduction, milk yield, carcass trait of Honamli goats and to make a comparison between the anatomic characteristics of this breed and Hair goats. Project of TUBITAK, No: 112R031, 2016.

19. Atay O, Gökdal O, Ozugur AK, et al. Relationships between udder measurements and milk yield characteristics of Hair goats in rural conditions. Paper presented at: 7st National Animal Ssience Scientific Congress; September 14-16, 2011; Adana-Turkey.

20. Erol H, Ozdemir P, Odabas E, et al. Investigation of various production traits of Angora goat herds kept as gene source both ex situ and in situ conditions. Lalahan Hay Araşt Enst Derg. 2017;57(1):1-12.

21. Cedden F, Kor A, Keskin S. Somatic cell counts in goat milk during late lactation period and its relationship with milk yield, age and some udder measurements. YYU J Agr Sci. 2002;12(2):63-67. 